\title{
Epigenetics of Glioblastoma Multiforme
}

Rasime $\mathbf{K}^{*}$

Department of Medical Genetics, Faculty of Medicine, Near East University, Nicosia, Turkish Republic of Northern Cyprus

*Corresponding author: Rasime K, Department of Medical Genetics, Faculty of Medicine, Near East University, Nicosia, Turkish Republic of Northern Cyprus, Tel: +90 392675 1000; Ext: 3017; Fax: +90 39267510 90; Email: kalkanr@yahoo.com

Received date: January 22, 2014; Accepted date: June 25, 2015; Published date: June 30, 2015

Copyright: (2015 Rasime K. This is an open-access article distributed under the terms of the Creative Commons Attribution License, which permits unrestricted use, distribution, and reproduction in any medium, provided the original author and source are credited.

\begin{abstract}
Aberrations in the epigenetic machinery of the genome may results the inactivation of critical genes and the epigenetic changes are important mechanisms in the evolution of malignancies that not only contributes to tumorigenesis but may also precede genetic changes. Several epigenetic mechanisms have been observed in glioblastomas including DNA hyper-methylation of genes, histone modifications including methylation and acetylation, nucleosomal rearrangement and dysregulation of noncoding RNA expression have been shown to play a critical role in the biology of glioblastomas and to contribute to the clinical outcome. This review examines the general role of epigenetic changes in the malignant process and focuses on the known epigenetic changes and development of new therapeutic strategies against these malignancies.
\end{abstract}

Keywords: Glioblastoma; Epigenetics; Methylation

\section{Introduction}

Glioblastoma multiforme (GBM) is the most malignant forms of astrocytoma [1,2]. It is the most common primary brain tumour in adults, accounting for $80 \%$ of all high-grade primary central nervous system (CNS) neoplasms [3,4]. GBM can be sub-classified into multiple groups, indistinguishable by histological appearance, but correlating with molecular-genetic factors as well as key clinical variables such as patient age and tumour location. The gliomagenesis is multistep process and the combination of acquired genetic abnormalities plays a crucial role in the progression of the tumour. The glioblastoma has been preceded by a number of recognizable lesions and the timing of these changes has been documented to be associated with the occurrence of abnormalities in different tumour suppressor genes.

Median survival of GBM is approximately 12 to 14 months and the prognosis and survival of patients remains poor $[1,3,4]$. The current treatments of these tumours are surgical resection, irradiation and conventional chemotherapy generally inadequate and unable to overcome the malignant biology of the tumour $[5,6]$. The currently used drugs are nonspecific and non-targeted nature (e.g., alkylating agents, DNA topoisomerase inhibitors, spindle poisons) $[7,8]$ so the result of conventional chemotherapy is poor. The conventional forms of treatment for GBM are not predicated on the biology of the malignant phenotype. Due to the insufficiency of conventional therapeutic approaches for GBM, new treatment modalities must be developed that have a more molecular, "targeted" mechanism of action [9-14].

The genome of glioblastoma cells shows global hypo-methylation with specific areas of hypermethylation. Hypermethylation mostly occurs at the promoter $\mathrm{CpG}$ islands of genes that are associated with tumour suppression. This pattern has been associated with increased genetic instability, silencing of tumour suppressor genes such as TP53 and PTEN, and activation of oncogenes. The Cancer Genome Atlas project has identified a glioma $\mathrm{CpG}$ island methylation phenotype that correlated with younger age, a proneural gene expression profile, and longer overall survival in glioblastoma patients [15].

The remarkable advances in defining the GBM cell of origin have been paralleled by insights into the genetic and epigenetic underpinnings of this disease.

EGFR, PDGFR, PI3K, NF1, TP53, Rb, IDH1/IDH2 and FGFR are only a few GBM mutational "drivers," these are important mutations to understand the genomic networks misregulated in GBM. The Cancer Genome Atlas (TCGA) Research Network proposed "proneural" "mesenchymal" "classical" and "neural" as a four subtypes of GBM based on genomic profiling of hundreds of human samples. Proneural GBMs show altered expression of PDGFRA, IDH1, TP53, PTEN mutation and CDKN2A loss. Mesenchymal GBMs have deletion of NF1, mutation of TP53 and PTEN, and loss of CDKN2A. Classical GBMs carried EGFR amplification and lack of PTEN, and CDKN2A and the neural GBMs show a strong expression of neuron markers and genes associated with neuron projection and axon and synaptic transmission.

microRNAs and long non-coding RNA are affect gene expression through regulation of mRNA stability and transcription regulation. MicroRNAs are non-coding RNAs, which bind to microRNA response elements (MREs) in target mRNAs. miRNA is bind to the RISC complex (RNA-induced silencing complex), the miRNA/RISC complex binds the target mRNA, thereby modulating its stability. miRNAs dysregulated in glioma include miR10b, which is expressed in glioma tumours and stem cells and miR10b controls GBM cell and stem cell cycle traverse and is correlated with poor prognosis (http:// tcga-data.nci.nih. gov/tcga) [16].

Long non-coding RNAs (lncRNAs), which are control global gene repression. IncRNAs control multiple tumour suppressor proteins, oncogenes and modulate transcription, regulate post-transcriptional RNA processing, influence translation, alter DNA methylation and chromatin architecture through local (cis) and long distance (trans) mechanisms. lncRNAs may play a crucial role in GBM development and progression. IncRNA MEG3 has been implicated in glioma cell 
Page 2 of 6

proliferation and MEG3 expression is associated with differential methylation[16].

Genetic changes are well recognized in GBM and include alterations in the $\mathrm{Rb} / \mathrm{p} 16$ pathway ( $>90 \%$ ) loss of heterozygosity of $10 \mathrm{q}$ (70\%), EGFR amplification (34\%) and TP53 mutations (31\%) [17]. Epigenetic changes, also known to occur in GBM, are less well characterized and their relation to clinical outcome is as yet uncertain.

There are several different components for genetic and epigenetic alterations that may be required for driving the unmitigated proliferation of glioblastoma cells.

\section{Epigenetic Alterations and Glioblastoma Progression}

Cancer progression is the current concept of cancer epigenetics. Epigenetics is the mitotically heritable changes in gene expression that are not due to changes in the DNA sequences. One of the best characterized epigenetic markers is DNA methylation. The initial finding of global hypo-methylation/hypermethylation in tumours [18] and the more recent discovery of induced inactivation of microRNA genes [18-20].

The DNA methylation occurs in cytosines that precede the guanines and these are commonly called dinucleotide CpGs. CpGs are not randomly distributed in the genome, but instead are present as CpG-rich regions referred to as CpG islands, which occur at the 5' end of the genes. These islands are usually not methylated in the normal tissues. DNA methylation occurs due to the chemical modifications of the histone proteins. Histones not only package DNA but also act as regulators of gene expression.

Locus-specific hypermethylation is frequent in GBM. The hypermethylation status of tumour suppressor genes is contributed to the pathogenesis of glioblastoma. In GBM, CpG island promoter hypermethylation occurs at genes to tumorigenesis and tumour progression, including tumour suppressors (RB1, EMP3, RASSF1A and BLU) [21], cell cycle regulation (p16INK4a and p15INK4b), DNA repair (MGMT, MLH1), apoptosis (DAPK, TIMP3 and CDH1), angiogenesis, invasion and drug resistance [22]. Patterns of DNA methylation in primary GBM specimens have been catalogued and include disruptions at many novel candidate tumour suppressors, such as the cell motility regulator TES (testis-derived transcript) as well as many polycomb repressor complex 2 (PRC2) target genes [23].

Hypermethylation of the tumour suppressor $\mathrm{Rb}$ [24] was soon followed by the discovery that this mechanism also contributed to the suppression of other genes such as VHL [25], p16INK4a [24], and BRCA1 (breast cancer susceptibility gene 1) [26].

Increased expression of EZH2 correlates with poor prognosis in GBM. The EZH2 genetic inhibition or pharmacologic inhibition can prevent self-renewal and tumourigenicity of glioblastoma cancer stem cells [21]. Caren et al was shown that the BMPR1B down-regulated in about $20 \%$ of primary glioblastoma tumours, and this is correlated with increased promoter DNA methylation [21].

The hypermethylation of genes might act as a predictor to treatment response and the MGMT (O6-methylguanine DNA methyltransferase) is the most studied DNA repair gene for the methylation-associated suppression of the DNA repair protein in glioblastomas [27]. MGMT essentially reverses addition of alkyl groups to the guanine base of the DNA making it prone to attack by alkylating agents [28]. Two studies showing that the hypermethylation of MGMT is an independent predictor for favorable prognosis in glioblastomas treated with either carmustine [29] or temozolomide [30].

Methylation of MGMT promoter was a correlative marker for response to alkylating agents; a recent clinical trial by Stupp et al. was established a new standard using chemoradiation with concurrent and adjuvant temozolomide for treatment of patients with newly diagnosed GBM, and the methylation status of MGMT promoter was correlated it with outcome [31].

Hegi et al. showed that methylated MGMT patients had an improved survival after chemoradiation than those with unmethylated MGMT, suggesting a direct predictive relationship between MGMT function and sensitivity to alkylating agents [30]. If improved outcome is seen in MGMT promoter methylated patients but who do not receive treatment with alkylating agents, this result suggest that a mechanism independent of MGMT-mediated resistance to alkylating agents could be responsible for this effect. Recent studies were showed MGMT methylation is not predictive marker in primary GBMs without any treatment applied patients [32].

Sato et al demonstrated that in stem-like glioblastoma cells the MGMT expression contributes to temozolomide resistance and a signalling pathway, MEK-ERK-MDM2-p53-MGMT, is operative in stem-like glioblastoma cells and plays a key role in the regulation of their MGMT expression. The temozolomide treatment combination with MEK targeting is effectively reduces tumorigenic potential of stemlike glioblastoma cells. This combination could also contribute to prevention of tumour recurrence [33].

Oberstadt et al showed significantly increased MGMT and ABCB1 promoter methylation in GBM tissues, but no significant association between overall survival of glioblastoma patients and MGMT or ABCB1 promoter methylation [34].

PTEN is the another critical tumour suppressor gene, the protein product for the PTEN is the functions as an inhibitor of PI3-kinase and inhibits the PI3K-Akt survival pathway, is associated with de novo GBM and less commonly with secondary GBM [35]. PTEN gene is inactivated by mutation in $20-40 \%$ of these tumours; loss of PTEN expression can occur in the absence of mutations, suggesting an epigenetic means of silencing. Baeza et al. reported PTEN promoter methylation in 35\% (22/77 tumors) of primary GBM [36]. Weincke et al. reported methylation of a region approximately $2.4 \mathrm{~kb}$ upstream of the start site of PTEN which was frequently seen in secondary GBM but not in de novo GBM [37]. This finding was seen in low-grade astrocytoma and suggesting that PTEN promoter methylation was an early event in the evolution of these tumors progression to glioblastoma.

Hypermethylation of TMS1/ASC (target of methylation-induced silencing-1), an adaptor protein that transduces death receptor apoptosis signals and has a role in inflammation, has been reported in $21 \%$ of GBM by Martinez et al.; the particular significance is the observation that $57 \%$ of long-term survivors ( $>3$ years) had promoter methylation of TMS1/ASC [38].

Stone et al. also reported TMS1/ASC promoter methylation in 43\% of GBM but did not report association with survival [39]. Folz et al. were identified BEX1 and BEX2 as tumour suppressor genes, the promoters of which are hypermethylated in GBM but not in nontumor brain tissue and demonstrated that overexpression of these genes in glioma xenografts suppressed tumor growth [40]. 
Knobbe et al. reported epigenetic silencing of carboxyl-terminal modulator protein (CTMP) gene, the products of the CTMP acts as a negative regulator of Akt, in $40 \%$ of GBMs tested, and the aberrant expression of CTMP in dysregulation of survival pathways in GBM [41]. The SLIT gene family is important in migration and axonal guidance and the the epigenetic inactivation of this genes were reported by Dallol et al. the promoter hypermethylation of the SLIT2 in $59 \%$ of glioma tissue samples tested, whereas in non-tumour brain tissue, the promoter was fully unmethylated [42]. This did not correlate with clinical outcome, but in a subset of tumours, the methylated tumours also had lower protein expression.

Watanabe et al. reported promoter methylation of the P73 in 5 of 28 (18\%) GBM patients but not in low-grade or anaplastic astrocytoma [43].

The analysis of the methylation status of cytokine signalling (SOCS)1-2-3 in GBMs showed methylation of SOCS3 promoter to be significantly associated with an unfavorable clinical outcome [44]. Zhou et al showed SOCS1, but not SOCS3 that was epigenetically silenced in GBM [45]. The N-myc downstreamregulated gene 2 (NDRG2) is a repressor of tumour cell proliferation, was found $\% 62$ hypermethyleted in primary GBM, but not in secondary or lower grade astrocytomas [46].

Schwatzentruber et al. showed that somatic mutations in the H3.3ATRX-DAXX chromatin-remodelling pathway frequently occur in paediatric GBMs and are associated with alternative lengthening of telomeres and genomic instability [47]. Another approach to discovery is to consider the epigenetic drivers of gliomagenesis. Strum et al. incorporated the mutational status of H3F3A and IDH1 with differences in global methylation patterns in GBMs to identify 6 distinct epigenetic subgroups, which correlate with distinct clinical characteristics [48].

Doxorubicin (DOX) is a chemotherapeutic drug for cancer treatment. DOX acts as a topoisomerase I poison by preventing DNA replication and also DOX can be involved in epigenetic regulation of gene transcription through downregulation of DNA methyltransferase 1 (DNMT1) then reactivation of DNA methylation-silenced tumour suppressor genes in glioblastoma (GBM) [49].

Glioma pathogenesis-related protein 1 (RTVP-1) has oncogenic features in glioblastoma (GBM; World Health Organization class IV) and highly expressed in GBMs [50]. The RTVP-1 promoter was hypomethylated in GBM. Hypermethylation of the RTVP-1 promoter was associated with improved overall survival in GBMs and the overexpression of this gene was associated with increased proliferation, enhanced invasion and inhibition of apoptosis [51,52].

microRNA-137 (mir-137) was described as a regulator of RTVP-1 gene. Downregulation of mir-137 contributes to the high expression of RTVP-1 in glioblastoma. The current study describes further loss of regulatory control of RTVP-1 in GBM by promoter methylation. Unlike GBM, RTVP-1 was hypermethylated in oligodendroglioma, another astrocytic tumor. While this may indicate specificity of RTVP-1 hypomethylation in GBM, it may also reflect general hypermethylation observed in oligodendrogliomas [53-55].

Besides inactivation of DNA repair genes, DNA methylation analyses showed that silencing of negative regulators of mitogenic pathways or activators of apoptosis is common in cancer showing tumor type-specific patterns. In GBM the WNT pathway may be activated through promoter methylation of negative regulators such as the WNT inhibitory factor 1 , the family of secreted frizzled-related proteins (sFRPs), dickkopf (DKK), and naked (NKDs) [56,57]. Another example is the Ras pathway that in a subset of GBM is deregulated by silencing of the negative regulators Ras association (RalGDS/AF-6) domain family members RASSF1A and RASSF10 $[58,59]$.

GBMs showed low levels of NPTX2 transcripts and the overexpression of NPTX2 gene induced apoptosis, inhibited proliferation and anchorage-independent growth, and rendered glioma cells chemosensitive. Furthermore, NPTX2 repressed NF-кB activity by inhibiting AKT through a p53-PTEN-dependent pathway, thus explaining the hypermethylation and downregulation of NPTX2 in NF- $\kappa B$-activated high-risk GBMs. Prosurvival NF- $\kappa B$ pathway activation characterized high-risk patients with poor prognosis, indicating it to be a therapeutic target [60].

Global hypomethylation occurs at a high frequency (80\%) in primary glioblastomas and the level of hypomethylation varies between GBMs, ranging from near normal brain levels to approximately $50 \%$ of normal [22].

\section{Somatic Mutations Causes DNA Methylation}

Promoter-associated $\mathrm{CpG}$ islands DNA hypermethylation of tumour suppressor and DNA repair genes, has been the most studied epigenetic alteration in human neoplasia $[61,62]$. Improvement of technology in the last few years allows comprehensive analysis of genome-wideDNA methylation on high-throughput platforms. Largescale analysis in GBM on aberrant DNA methylation at CpG sites has unraveled a plethora of genes that are affected [55]. Widespread CpG island promoter methylation, also referred to as the CpG island methylator phenotype (CIMP), was first identified by Toyata et al [63] and has been extensively studied in many cancer types. The first two studies by Noushmehr et al [55] and Turcan et al [64] showed that glioblastomas with a hypermethylator phenotype are associated with somatic mutations in isocitrate dehydrogenase-1 [55,64] and that somatic mutations in IDH1, IDH2, as well as loss-of-function mutations in ten-eleven translocation (TET)-methylcytosine dioxygenase-2 (TET2) establish a hypermethylation phenotype in leukemia [65]. As we know about that the glioblastomas have a two distinct subtypes which are primary and secondary GBM. These are the two distinct disease entities which are the genetic and the epigenetic background of these tumors are highly variable. From a clinical and biological perspective, two main subtypes of this malignancy can be distinguished: primary (or de novo) glioblastoma develops without the presence of any precursor neoplastic lesion and manifests after a short clinicalhistory; secondary glioblastoma develops from lower grade tumors [66]. Mutations of IDH1 are rare in primary GBM (10\%) and frequent in secondary GBM (80\%) [67]. Than the primary glioblastomas which are showed low frequencies of IDH1 mutations and G-CIMP have a different pathogenetic/epigenetic origin than secondary glioblastoma and should be classified separately. The correlation of the neomorphic IDH1/2 mutants with a DNA methylator phenotype was also observed in acute myeloid leukemia (AML). This provided an important mechanistic link, together with the fact that IDH1/2 mutations in leukemia were exclusive with tet oncogene family member 2 ( TET2 ) mutations [65,68]. Moreover, a high frequency of IDH1 mutations indicates a link between metabolic alterations and epigenetic modification in these tumors [15]. A mutation in IDH1 inhibits DNA demethylation and causes accumulation of the methylated DNA. IDH are NAD+ and NADP+- 
Page 4 of 6

dependent enzymes that catalyze the tricarboxylic acid (TCA) cycle, and mutations in IDH1 produce a metabolite called 2hydroxyglutarate (2-HG) to accumulate. The accumulated 2-HG impairs the activity of ten-eleven translocation (TET) methylcytosine dioxygenase, which results in DNA hypermethylation. IDH1 mutation may result in G-CIMP through inhibition of the TET-mediated production of 5-hydroxymethylcytosine $(5 \mathrm{hmC})$, which is a primary mode of DNA demethylation [69].

Exome sequencing studies showed that at least one somatic mutation in genes which are DNA methylation (isocitrate dehydrogenase [IDH] 1, IDH2), histone modification (mixed lineage leukaemia 2 [MLL2], MLL3, MLL4, Enhancer of zeste 2 [EZH2] and histone deacetylase 2 [HDAC2]) and chromatin remodelling (athalassaemia/mental retardation syndrome X-linked [ATRX], deathdomain associated protein [DAXX], CREB binding protein [CREBBP] and SWI/SNF-related matrix-associated, actin-dependent regulator of chromatin A2 [SMARCA2]) associated with chromatin modification [70]. These mutations result in the impairment of DNA methylation, histone modification and nucleosome positioning, and are associated with aberrant gene expression [71,55]. The crosstalk between the genome and epigenome might suggest new molecular targets and possibilities for the treatment of GBM [69].

Methylation of K27 and K36 is also disrupted by elevated levels of the onco-metabolite 2-hydroxyglutarate (2-HG) resulting from gainof-function mutations in $I D H 1[68,72]$, Which was previously shown to be associated with a distinct Glioma-CpG-Island Methylator Phenotype (G-CIMP) [55].

\section{Are Epigenetic-based therapies possible for GBM?}

Epigenetic modifications by its nature are reversible; therefore changes in the epigenome associated with cancer are potentially reversible. It opens up the possibility of using epigenetic drugs which may have a powerful impact on various cancers, including glioblastomas and the major unresolved problem for epigenetic therapy of cancer is target specificity [22]. Two demethylating agents, i.e., inhibitors of DNA methyltransferase (5-azacytidin and decitabin) were approved by the Food and Drug Administration (FDA) in the treatment of myelodysplastic syndrome and a few inhibitors of histone acetylases (vorinostat, romidepsin and panobinostat) are approved in the treatment of hematological malignancies, particularly in refractory or relapsed cutaneous T-cell lymphoma [73]. Other compounds are presently in clinical trials and the hypomethylating agents are also one of the few epigenetic therapies that have gained FDA approval for routine clinical use. Small-molecule inhibitors of histone demethylases are at various stages of development and emerging preclinical data show the therapeutic potential of compounds [74].

The DNMT inhibitor Decitabine (5-aza-2'-deoxycytidine) and the HDACi suberoylanilide hydroxamic acid (SAHA; Vorinostat) are the epigenetic-based therapies and only HDACi are in trials for GBM [22]. HDACi are hydroxymates (SAHA, TSA), cyclic peptides (depsipeptide), aliphatic acids (valproic acid, butyrate), and benzamides. No single HDACi is effective against all HDACs. HDACi cause increased acetylation of histone and non-histone proteins and can reactivate $\mathrm{p} 21$, which contributes to cell-cycle arrest. Noncancerous cells are more resistant to the effects of HDACi $[75,76]$.

Epigenetic therapies approved by FDA for leukemia and DNA demethylating agents and HDAC inhibitors, and combinations have been tested in clinical trials [77]. In glioblastoma HDAC inhibitors have entered clinical trials (http://clinicaltrials.gov), while demethylating agents have not been considered [78]. Demethylating agents such as 5-Aza-cytidine or 5-Aza-2 $\$$ - deoxcytidine lock DNMT enzymes on to the DNA, thereby inhibiting further DNA methylation. Consequently, demethylating agents require cell division for activity, hence targeting rapidly dividing cells. Due to their unspecific mechanism, demethyling agents may lead to reexpression not only of tumor suppressor genes but also of oncogenes. The HDAC inhibitor vorinostat (SAHA) has shown modest benefit as single agent in a phase II trial for recurrent GBM [79]. The other treatment choise was TMZ and the methylated MGMT promoter sensitizes the tumors to alkylating agents and that the alkylating agent TMZ is part of the current standard of care for GBM [78]. The treatment of GBM patients with valproic acid as antiepileptic drug has shown a survival advantage in combined chemoradiotherapy [80]. Valproic acid is considered to have weak HDAC inhibitor properties and is currently tested in a phase 2 trial for newly diagnosed GBM in combination with standard chemoradiotherapy (NCT00302159). Entinostat, panobinostat phenylbutyrate are the other HDAC inhibitors which are in clinical evaluation for recurrent high-grade gliomaor refractory pediatric brain tumors and neuroblastoma [77]. The AGI-5198 inhibitor identified through a high-throughput screen as a selective R132H-IDH1 inhibitor, induced demethylation of histone $\mathrm{H} 3 \mathrm{~K} 9 \mathrm{me} 3$ and expression of genes associated with gliogenic differentiation. Blockade of a mutant IDH1 impaired the growth of IDH1-mutant, but not IDH1wild-type, glioma cells [81].

\section{Conclusion}

Necrotic or apoptotic cancer cells releasing genomic DNA and the blood plasma in cancer patients contains DNA derived from these cells and aberrantly hypermethylated genes found in plasma could be one such type of biomarker. This is a less invasive method for biomarker detection in cancer patients. This type of analysis may provide clinically useful information about diagnosis, prognosis and follow-up post-therapy. There is a significant amount of tumour DNA in the plasma of high-grade glioma patients, and in $60 \%$ of patients the same methylated promoters (p16INK4a, MGMT, p73, and RARbeta) could be detected in both tumour and plasma DNA [82].

Primary and secondary glioblastoma is a distinct disease subtypes and affecting patients of different age and developing through different genetic pathway. Based on these two subgroups of GBM we may use this different methylation pattern of genes to distinguish between subtypes and aberrant DNA methylation of tumour suppressor genes and oncogenes reported in GBM. The summerized studies mentioned emphasize the relevance of epigenetic gene silencing as a mechanism by which critical biologic pathways are altered in GBM. Several genes discussed above have been identified singly or by genome-wide methylation studies to be epigenetically inactivated. The relationship of aberrant hypermethylation of promoters to the biology of GBM and the prognosis of patients with these tumours is complex and remains to be fully elucidated; it is possible that a more fundamental defect in the epigenetic machinery that regulates promoter methylation may be responsible for the epigenetic changes that cause inactivation of several cancer-related genes and the identification of IDH1/2 mutations in GBM suggests that metabolic pathways may be attractive targets for GBM. 


\section{References}

1. Newton HB (1994) Primary brain tumors: review of etiology, diagnosis and treatment. Am Fam Physician 49: 787-797.

2. Kleihues P, Louis DN, Scheithauer BW, Rorke LB, Reifenberger G, et al. (2002) The WHO classification of tumors of the nervous system. J Neuropathol Exp Neurol 61: 215-225.

3. Davis FG, McCarthy BJ (2001) Current epidemiological trends and surveillance issues in brain tumors. Expert Rev Anticancer Ther 1: 395-401.

4. Wrensch M, Minn Y, Chew T, Bondy M, Berger MS (2002) Epidemiology of primary brain tumors: current concepts and review of the literature. Neuro Oncol 4: 278-299.

5. Fine HA, Dear KB, Loeffler JS, Black PM, Canellos GP (1993) Metaanalysis of radiation therapy with and without adjuvant chemotherapy for malignant gliomas in adults. Cancer 71: 2585-2597.

6. Stewart LA (2002) Chemotherapy in adult high-grade glioma: a systematic review and meta-analysis of individual patient data from 12 randomised trials. Lancet 359: 1011-1018.

7. Newton HB, Turowski RC, Stroup TJ, McCoy LK (1999) Clinical presentation, diagnosis, and pharmacotherapy of patients with primary brain tumors. Ann Pharmacother 33: 816-832.

8. Newton HB (2006) Clinical pharmacology of brain tumour chemotherapy In: Newton HB (ed.) Handbook of brain tumor chemotherapy. Elsevier, pp. 21-43.

9. Mischel PS, Cloughesy TF (2003) Targeted molecular therapy of GBM. Brain Pathol 13: 52-61.

10. Newton HB (2003) Molecular neuro-oncology and development of targeted therapeutic strategies for brain tumors. Part 1: Growth factor and Ras signaling pathways. Expert Rev Anticancer Ther 3: 595-614.

11. Newton HB (2004) Molecular neuro-oncology and development of targeted therapeutic strategies for brain tumors. Part 2: PI3K/Akt/PTEN, mTOR, SHH/PTCH and angiogenesis. Expert Rev Anticancer Ther 4: 105-128.

12. Newton HB (2005) Molecular neuro-oncology and the development of targeted therapeutic strategies for brain tumors. Part 5: apoptosis and cell cycle. Expert Rev Anticancer Ther 5: 355-378.

13. Rich JN, Bigner DD (2004) Development of novel targeted therapies in the treatment of malignant glioma. Nat Rev Drug Discov 3: 430-446.

14. Tremont-Lukats IW, Gilbert MR (2003) Advances in molecular therapies in patients with brain tumors. Cancer Control 10: 125-137.

15. Thon N, Kreth S, Kreth FW (2013) Personalized treatment strategies in glioblastoma: MGMT promoter methylation status. Onco Targets Ther 6 1363-1372.

16. Cancer Genome Atlas Research Network (2008) Comprehensive genomic characterization defines human glioblastoma genes and core pathways. Nature 455: 1061-1068.

17. Amatya VJ, Naumann U, Weller M, Ohgaki H (2005) TP53 promoter methylation in human gliomas. Acta Neuropathol 110: 178-184.

18. Brower JV, Clark PA, Lyon W, Kuo JS (2014) MicroRNAs in cancer: glioblastoma and glioblastoma cancer stem cells. Neurochem Int 77: 68-77.

19. Saito Y, Liang G, Egger G, Friedman JM, Chuang JC, et al. (2006) Specific activation of microRNA-127 with downregulation of the proto-oncogene BCL6 by chromatin-modifying drugs in human cancer cells. Cancer Cell 9: 435-443.

20. Lujambio A, Ropero S, Ballestar E, Fraga MF, Cerrato C, et al. (2007) Genetic unmasking of an epigenetically silenced microRNA in human cancer cells. Cancer Res 67: 1424-1429.

21. Carén H, Pollard SM, Beck S (2013) The good, the bad and the ugly: epigenetic mechanisms in glioblastoma. Mol Aspects Med 34: 849-862.

22. Nagarajan RP, Costello JF (2009) Epigenetic mechanisms in glioblastoma multiforme. Semin Cancer Biol 19: 188-197.
23. Martinez R, Martin-Subero JI, Rohde V, Kirsch M, Alaminos M, et al. (2009) A microarray-based DNA methylation study of glioblastoma multiforme. Epigenetics 4: 255-264.

24. Greger V, Passarge E, Höpping W, Messmer E, Horsthemke B (1989) Epigenetic changes may contribute to the formation and spontaneous regression of retinoblastoma. Hum Genet 83: 155-158.

25. Herman JG, Latif F, Weng Y, Lerman MI, Zbar B, et al. (1994) Silencing of the VHL tumor-suppressor gene by DNA methylation in renal carcinoma. Proc Natl Acad Sci U S A 91: 9700-9704.

26. Herman JG, Baylin SB (2003) Gene silencing in cancer in association with promoter hypermethylation. N Engl J Med 349: 2042-2054.

27. Esteller M, Hamilton SR, Burger PC, Baylin SB, Herman JG (1999) Inactivation of the DNA repair gene O6-methylguanine-DNA methyltransferase by promoter hypermethylation is a common event in primary human neoplasia. Cancer Res 59:793-797.

28. Esteller M, Herman JG (2004) Generating mutations but providing chemosensitivity: the role of O6-methylguanine DNA methyltransferase in human cancer. Oncogene 23: 1-8.

29. Esteller M, Garcia-Foncillas J, Andion E, Goodman SN, Hidalgo OF (2000) Inactivation of the DNA-repair gene MGMT and the clinical response of gliomas to alkylating agents. N Engl J Med 343: 1350-1354.

30. Hegi ME, Diserens AC, Gorlia T, Hamou MF, de Tribolet N, et al. (2005) MGMT gene silencing and benefit from temozolomide in glioblastoma. N Engl J Med 352: 997-1003.

31. Stupp R, Mason WP, Bent MJ, Weller M, Fisher B, et al. (2005) European Organisation for Research and Treatment of Cancer Brain Tumor and Radiotherapy Groups; National Cancer Institute of Canada Clinical Trials Group. Radiotherapy plus concomitant and adjuvant temozolomide for glioblastoma. N Engl J Med 352: 987-996

32. Kalkan R, Atli Eİ, Özdemir M, Çiftçi E, Aydin HE, et al. (2015) IDH1 mutations is prognostic marker for primary glioblastoma multiforme but MGMT hypermethylation is not prognostic for primary glioblastoma multiforme. Gene 554: 81-86.

33. Sato A, Sunayama J, Matsuda K, Seino S, Suzuki K, et al. (2011) MEKERK signaling dictates DNA-repair gene MGMT expression and temozolomide resistance of stem-like glioblastoma cells via the MDM2p53 axis. Stem Cells 29: 1942-1951.

34. Oberstadt MC, Bien-Möller S, Weitmann K, Herzog S, Hentschel K, et al (2013) Epigenetic modulation of the drug resistance genes MGMT, $\mathrm{ABCB} 1$ and $\mathrm{ABCG} 2$ in glioblastoma multiforme. BMC Cancer 13: 617.

35. Phillips HS, Kharbanda S, Chen R, Forrest WF, Soriano RH, et al (2006) Molecular subclasses of high-grade glioma predict prognosis, delineate a pattern of disease progression, and resemble stages in neurogenesis. Cancer Cell 9:157-173.

36. Baeza N, Weller M, Yonekawa Y, Kleihues P, Ohgaki H (2003) PTEN methylation and expression in glioblastomas. Acta Neuropathol 106: 479-485.

37. Wiencke JK, Zheng S, Jelluma N, Tihan T, Vandenberg S, et al. (2007) Methylation of the PTEN promoter defines low-grade gliomas and secondary glioblastoma. Neuro Oncol 9: 271-279.

38. Martinez R, Schackert G, Esteller M (2007) Hypermethylation of the proapoptotic gene TMS1/ASC: prognostic importance in glioblastoma multiforme. J Neurooncol 82: 133-139.

39. Stone AR, Bobo W, Brat DJ, Devi NS, Van Meir EG, et al. (2004) Aberrant methylation and down-regulation of TMS1/ASC in human glioblastoma. Am J Pathol 165: 1151-1161.

40. Foltz G, Ryu GY, Yoon JG, Nelson T, Fahey J, (2006) Genome-wide analysis of epigenetic silencing identifies BEX1 and BEX2 as candidate tumor suppressor genes in malignant glioma. Cancer Res 66: 6665-6674.

41. Knobbe CB, Reifenberger J, Blaschke B, Reifenberger G (2004) Hypermethylation and transcriptional downregulation of the carboxylterminal modulator protein gene in glioblastomas. J Natl Cancer Inst 96: 483-486 
42. Dallol A, Krex D, Hesson L, Eng C, Maher ER, et al. (2003) Frequent epigenetic inactivation of the SLIT2 gene in gliomas. Oncogene 22: 4611-4616.

43. Watanabe T, Huang $\mathrm{H}$, Nakamura $\mathrm{M}$, Wischhusen J, Weller $\mathrm{M}$, et al. (2002) Methylation of the p73 gene in gliomas. Acta Neuropathol 104: 357-362.

44. Martini M, Pallini R, Luongo G, Cenci T, Lucantoni C, et al. (2008) Prognostic relevance of SOCS3 hypermethylation in patients with glioblastoma multiforme. Int J Cancer 123: 2955-2960.

45. Zhou H, Miki R, Eeva M, Fike FM, Seligson D, et al. (2007) Reciprocal regulation of SOCS 1 and SOCS3 enhances resistance to ionizing radiation in glioblastoma multiforme. Clin Cancer Res 13: 2344-2353.

46. Tepel M, Roerig P, Wolter M, Gutmann DH, Perry A, et al. (2008) Frequent promoter hypermethylation and transcriptional downregulation of the NDRG2 gene at $14 \mathrm{q} 11.2$ in primary glioblastoma. Int J Cancer 123: 2080-2086.

47. Schwatzentruber J, Korshunov A, Liu XY, Jones DT, Pfaff E et al (2012) Driver mutations in histone H3.3 and chromatin remodelling genes in paediatric glioblastoma. Nature 482: 226-231.

48. Strum D, Witt H, Hovestadt V, Khuong-Quang DA, Jones DT et al (2012) Hotspot mutations in H3F3A and IDH1 define distinct epigenetic and biological subgroups of glioblastoma. Cancer Cell 22: 425-437.

49. Zhang S, Han L, Wei J, Shi Z, Pu P, Zhang J, Yuan X, Kang C (2015) Combination treatment with doxorubicin and microRNA-21 inhibitor synergistically augments anticancer activity through upregulation of tumor suppressing genes. Int J Oncol 46: 1589-600.

50. Jacoby E, Yalon M1, Leitner M, Cohen ZR, Cohen Y, et al. (2014) Related to testes-specific, vespid and pathogenesis protein-1 is regulated by methylation in glioblastoma. Oncol Lett 7: 1209-1212.

51. Murphy EV, Zhang Y, Zhu W (1995) The human glioma pathogenesis related protein is structurally related to plant pathogenesis related proteins and its gene is expressed specifically in brain tumors. Gene 159: 131-135.

52. Xiang C, Sarid R, Cazacu S, Finniss S, Lee HK, et al. (2007) Cloning and characterization of human RTVP-1b, a novel splice variant of RTVP-1 in glioma cells. Biochem Biophys Res Commun 362: 612-618.

53. Dong SM, Pang JC, Poon WS, Hu J, To KF, et al. (2001) Concurrent hypermethylation of multiple genes is associated with grade of oligodendroglial tumors. J Neuropathol Exp Neurol 60: 808-816.

54. Wolter M, Reifenberger J, Blaschke B (2001) Oligodendroglial tumors frequently demonstrate hypermethylation of the CDKN2A (MTS1, p16INK4a), p14ARF, and CDKN2B (MTS2, p15INK4b) tumor suppressor genes. J Neuropathol Exp Neurol 60: 1170-1180.

55. Noushmehr H, Weisenberger DJ, Diefes K, Phillips HS, Pujara K, et al. (2010) Identification of a CpG island methylator phenotype that defines a distinct subgroup of glioma. Cancer Cell 17: 510-522.

56. Lambiv WL, Vassallo I, Delorenzi M, Shay T, Diserens AC, et al. (2011) The Wnt inhibitory factor 1 (WIF1) is targeted in glioblastoma and has a tumor suppressing function potentially by induction of senescence. Neuro Oncol 13: 736-747.

57. Götze S, Wolter M, Reifenberger G, Müller O, Sievers S (2010) Frequent promoter hypermethylation of Wnt pathway inhibitor genes in malignant astrocytic gliomas. Int J Cancer 126: 2584-2593.

58. Horiguchi K, Tomizawa $\mathrm{Y}$, Tosaka M, Ishiuchi S, Kurihara H, et al. (2003) Epigenetic inactivation of RASSF1A candidate tumor suppressor gene at 3p21.3 in brain tumors. Oncogene 22: 7862-7865.

59. Hill VK, Underhill-Day N, Krex D, Robel K, Sangan CB, et al. (2011) Epigenetic inactivation of the RASSF10 candidate tumor suppressor gene is a frequent and an early event in gliomagenesis. Oncogene 30: 978-989.

60. Shukla S, Pia Patric IR, Thinagararjan S, Srinivasan S, Mondal B et al. (2013) A DNA methylation prognostic signature of glioblastoma: identification of NPTX2-PTEN-NF-?B nexus. Cancer Res 73: 6563-6573.

61. Mack GS (2006) Epigenetic cancer therapy makes headway. J Natl Cancer Inst 98: 1443-1444.
62. Esteller M (2008) Epigenetics in cancer. N Engl J Med 358: 1148-1159.

63. Toyota M, Ahuja N, Ohe-Toyota M, Herman JG, Baylin SB, et al. (1999) CpG island methylator phenotype in colorectal cancer. Proc Natl Acad Sci U S A 96: 8681-8686.

64. Turcan S, Rohle D, Goenka A, Walsh LA, Fang F, et al. (2012) IDH1 mutation is sufficient to establish the glioma hypermethylator phenotype. Nature 483: 479-483.

65. Figueroa ME, Abdel-Wahab O, Lu C, Ward PS, Patel J, et al. (2010) Leukemic IDH1 and IDH2 mutations result in a hypermethylation phenotype, disrupt TET2 function, and impair hematopoietic differentiation. Cancer Cell 18: 553-567.

66. Pojo M, Costa BM (2011) Molecular hallmarks of gliomas. In: Garami M (ed.) Molecular Targets of CNS Tumors. In Tech, pp. 177-200.

67. Håvik AB, Brandal P, Honne H, Dahlback HS, Scheie D, et al. (2012) MGMT promoter methylation in gliomas-assessment by pyrosequencing and quantitative methylation-specific PCR. J Transl Med 10: 36.

68. Xu W, Yang H, Liu Y, Yang Y, Wang P, et al. (2011) Oncometabolite 2hydroxyglutarate is a competitive inhibitor of $\alpha$-ketoglutarate-dependent dioxygenases. Cancer Cell 19: 17-30.

69. Kondo Y, Katsushima K, Ohka F, Natsume A, Shinjo K (2014) Epigenetic dysregulation in glioma. Cancer Sci 105: 363-369.

70. Brennan CW, Verhaak RG, McKenna A, Campos B, Noushmehr H, et al. (2013) The somatic genomic landscape of glioblastoma. Cell 155: 462-477.

71. Dawson MA, Kouzarides T (2012) Cancer epigenetics: from mechanism to therapy. Cell 150: 12-27.

72. Chowdhury R, Yeoh KK, Tian YM, Hillringhaus L, Bagg EA, et al. (2011) The oncometabolite 2-hydroxyglutarate inhibits histone lysine demethylases. EMBO Rep 12: 463-469.

73. Maleszewska M, Kaminska B (2013) Is glioblastoma an epigenetic malignancy? Cancers (Basel) 5: 1120-1139.

74. Spiller SE, Ravanpay AC, Hahn AW, Olson JM (2006) Suberoylanilide hydroxamic acid is effective in preclinical studies of medulloblastoma. J Neurooncol 79: 259-270.

75. Yin D, Ong JM, Hu J, Desmond JC, Kawamata N, et al. (2007) Suberoylanilide hydroxamic acid, a histone deacetylase inhibitor: effects on gene expression and growth of glioma cells in vitro and in vivo. Clin Cancer Res 13: 1045-1052.

76. Gui CY, Ngo L, Xu WS, Richon VM, Marks PA (2004) Histone deacetylase (HDAC) inhibitor activation of p21WAF1 involves changes in promoter-associated proteins, including HDAC1. Proceedings of the National Academy of Sciences of the United States of America 101: 1241-1246.

77. Kelly TK, De Carvalho DD, Jones PA (2010) Epigenetic modifications as therapeutic targets. Nat Biotechnol 28: 1069-1078.

78. Stupp R, Hegi ME, Mason WP (2009) Effects of radiotherapy with concomitant and adjuvant temozolomide versus radiotherapy alone on survival in glioblastoma in a randomised phase III study: 5-year analysis of the EORTC-NCIC trial. Lancet Oncol 10: 459-66.

79. Galanis E, Jaeckle KA, Maurer MJ, Reid JM, Ames MM, et al. (2009) Phase II trial of vorinostat in recurrent glioblastoma multiforme: a north central cancer treatment group study. J Clin Oncol 27: 2052-2058.

80. Weller M, Gorlia T, Cairncross JG, van den Bent MJ, Mason W, et al. (2011) Prolonged survival with valproic acid use in the EORTC/NCIC temozolomide trial for glioblastoma. Neurology 77: 1156-1164.

81. Rohle D, Popovici-Muller J, Palaskas N, Turcan S, Grommes C, et al. (2013) An inhibitor of mutant IDH1 delays growth and promotes differentiation of glioma cells. Science 340: 626-630.

82. Weaver KD, Grossman SA, Herman JG (2006) Methylated tumorspecific DNA as a plasma biomarker in patients with glioma. Cancer Investigatio 24: 35-40. 